\title{
EFFECTS OF SMOOTH, MEDIUM SMOOTH AND MEDIUM RECONSTRUCTION KERNELS ON IMAGE QUALITY IN THREE-PHASE CT OF LIVER
}

\author{
NUR AMALINA AMERUDDIN, SULAIMAN MD DOM and MOHD HAFIZI MAHMUD* \\ Centre for Medical Imaging Studies, Faculty of Health Sciences, \\ Universiti Teknologi MARA Selangor, Puncak Alam Campus, \\ 42300 Bandar Puncak Alam, Selangor, Malaysia \\ *E-mail: mhafizi@uitm.edu.my
}

Accepted 16 September 2021, Published online 30 November 2021

\begin{abstract}
Reconstruction kernel is one of the parameters that affects the computed tomography (CT) image quality. This study aimed to evaluate the effects of applying three different reconstruction kernels on image quality in 3-phased CT of the liver. A total of 63 CT liver images including normal liver $(n=43)$ and liver lesion $(n=20)$ were retrospectively reviewed. Smooth (B20f), medium smooth (B30f) and medium (B40f) reconstruction kernels were employed in the image reconstruction process. Mean attenuation, image noise, and signal-to-noise ratio (SNR) values from each kernel reconstruction were quantified and compared among those kernels using One Way Analysis of Variance (ANOVA) statistical analysis. Significant changes in image noise and SNR were observed in the normal liver $(p<0.001$, respectively) following the application of those reconstruction kernels. However, no significant changes in mean attenuation, image noise, and SNR were demonstrated in the liver lesion $(p>0.05)$. Application of smooth (B20f), medium smooth (B30f), and medium (B40f) kernel reconstructions would significantly affect the image noise and SNR in the normal liver of CT images instead of liver lesions. Hence, proper selection of reconstruction kernel is important in CT images reconstruction to improve precision in diagnostic CT interpretation.
\end{abstract}

Key words: CT liver, image quality, medium kernel, medium smooth kernel, smooth kernel

\section{INTRODUCTION}

Computed Tomography (CT) scan is the most common modality in diagnostic imaging for the abdominal area due to its excellent low-contrast image resolution (Seeram, 2015). Three-phase CT is a standard contrast-enhanced CT protocol for detection and visualization of the liver lesion, liver cirrhosis, and hepatic cellular carcinoma (Jensen et al., 2014). Reducing the radiation dose delivered by CT scans has become a major concern, particularly in abdominal imaging where the acquisition protocol may include three or four acquisition phases (Gervaise et al., 2014).

Over the past decade, image reconstruction in $\mathrm{CT}$ scans is rapidly evolving to produce an efficient yet accurate image reconstruction method while keeping radiation dose to a minimum (Sagara et

\footnotetext{
* To whom correspondence should be addressed.
}

al., 2010; Yu et al., 2016). Two major categories of CT image reconstruction techniques are analytic reconstruction and iterative reconstruction. Analytical reconstruction techniques are the formation of an image using a direct mathematical solution while iterative reconstruction methods use multiple steps of mathematical solution (Alsleem \& Davidson, 2013). Fourier transformation, simple back projection, back-projection filtering, and filtered backprojection (FBP) are the methods of analytical image reconstruction (Vardhanabhuti et al., 2013). FBP reconstruction technique is the most common method used in commercial CT scanners (Yu \& Leng, 2016). FBP processes the multiple acquired projections by back-projecting raw data to create an image and considering the measurements of $\mathrm{x}$-ray projections made at varying scanning angles (Patino et al., 2015). FBP assumes that the acquired projection data are fully free of noise (Sagara et al., 2010; Staniszewska \& Chrusciak, 2017). 
Correspondingly, decreasing radiation exposure is associated with increased noise in FBP reconstructed images (Scharf et al., 2017).

The reconstruction kernel of FBP is the software that provides filter variations during the filtering process (Jang et al., 2011) and it is one of the most important parameters that affects image quality ( $\mathrm{Yu}$ \& Leng, 2016). The reconstruction kernel provides different image properties according to the clinical task (Patino et al., 2015). Each kernel gives a different impact on the spatial resolution and noise of a CT image. A smoother kernel generates images with lower noise but with reduced spatial resolution, while a sharper kernel generates images with higher spatial resolution but increases the image noise (Yu \& Leng, 2016). Therefore, the selection of reconstruction kernels should be based on specific clinical applications (Jang et al., 2011).

The effects of FBP reconstruction kernels in CT liver have been mainly focused on the liver lesions in the previous literature (Jang et al., 2011; Yu \& Leng, 2016). However, those effects on the normal liver are not well reported. Therefore, this study is aimed to evaluate the effects of applying smooth, medium smooth, and medium reconstruction kernels on image quality in 3-phases CT of the liver in normal liver and liver lesions.

\section{MATERIALS AND METHODS}

\section{CT image retrieval}

This study was granted ethics approval from the Universiti Teknologi MARA (UiTM) Research Ethics Committee (UG/MR/118). A total of $63 \mathrm{CT}$ liver images (normal liver, $n=43$ and liver with lesion, $n=$ 20) of patients who underwent CT liver examination from December 2019 to July 2020 were retrospectively reviewed from the $\mathrm{CT}$ workstation at the Department of Radiology, Kuantan Medical Centre, Pahang. The age of patients ranged between 18 to 65 years old. Patients who had to undergo a three-phase CT liver examination were included while those from followup CT examination were excluded from this study.

CT images were acquired from three-phased CT liver examination using a 64-detector Somatom Definition AS + CT scanner (Siemens Healthcare). Transverse section images at the level of portal vein bifurcation were employed in this study, where both the right and left lobes of the liver were visualized. The images were reconstructed using three kernels: smooth (B20f), medium smooth (B30f), and medium (B40f) kernels following CT images acquisition.

\section{Image quality parameters quantification}

For normal liver images, a circular CT tool was used to select region-of-interest (ROI). Three circular $170 \mathrm{~mm}^{2}$ ROIs were placed on the left and right lobes (Figure 1). For images with the liver lesion, three circular ROIs with the size ranged $70-80 \mathrm{~mm}^{2}$ were placed in the identified lesion on each image (Figure 2). The ROIs were placed at the centre site of the primary lesions accordingly. The selection of ROIs was reviewed and validated by a senior radiologist.

Three image quality parameters were quantified on the reconstructed images including attenuation value, image noise, and signal-to-noise ratio (SNR). The mean attenuation values were generated from the average of the mean Hounsfield number of the

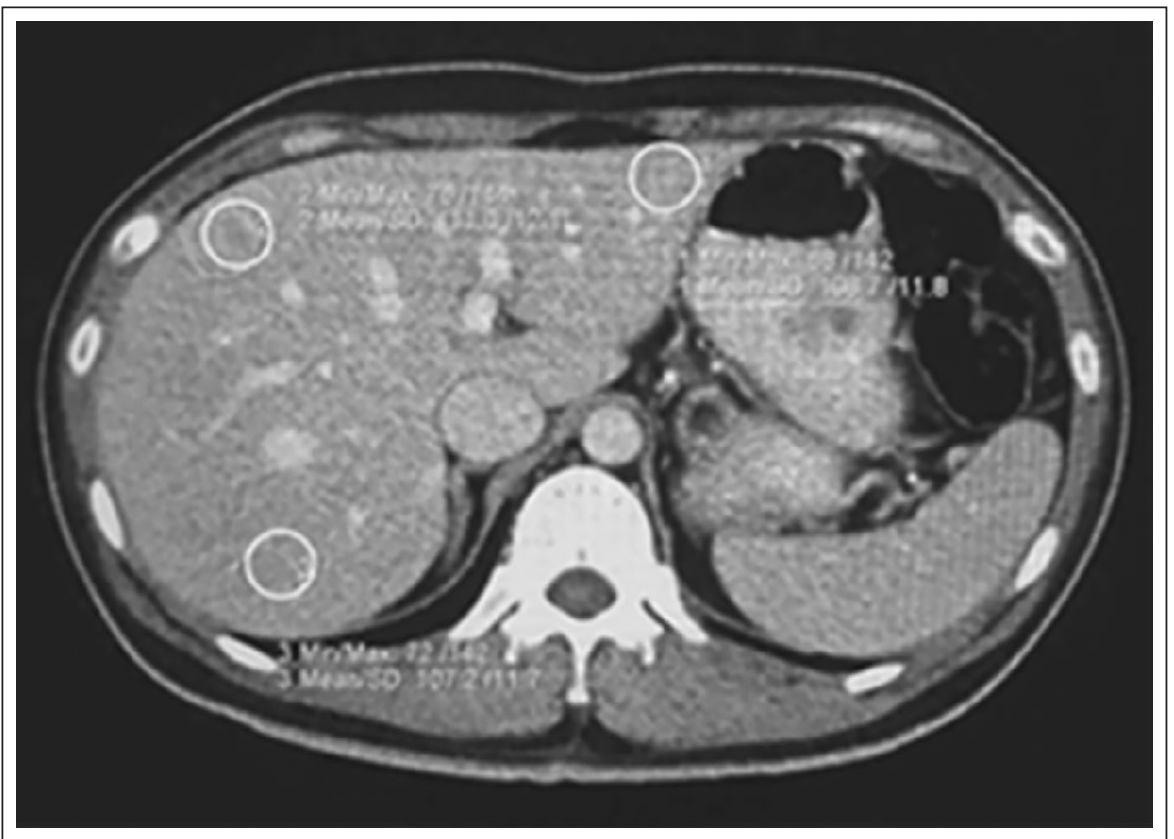

Fig. 1. The placement of ROIs on a CT image of normal liver. 


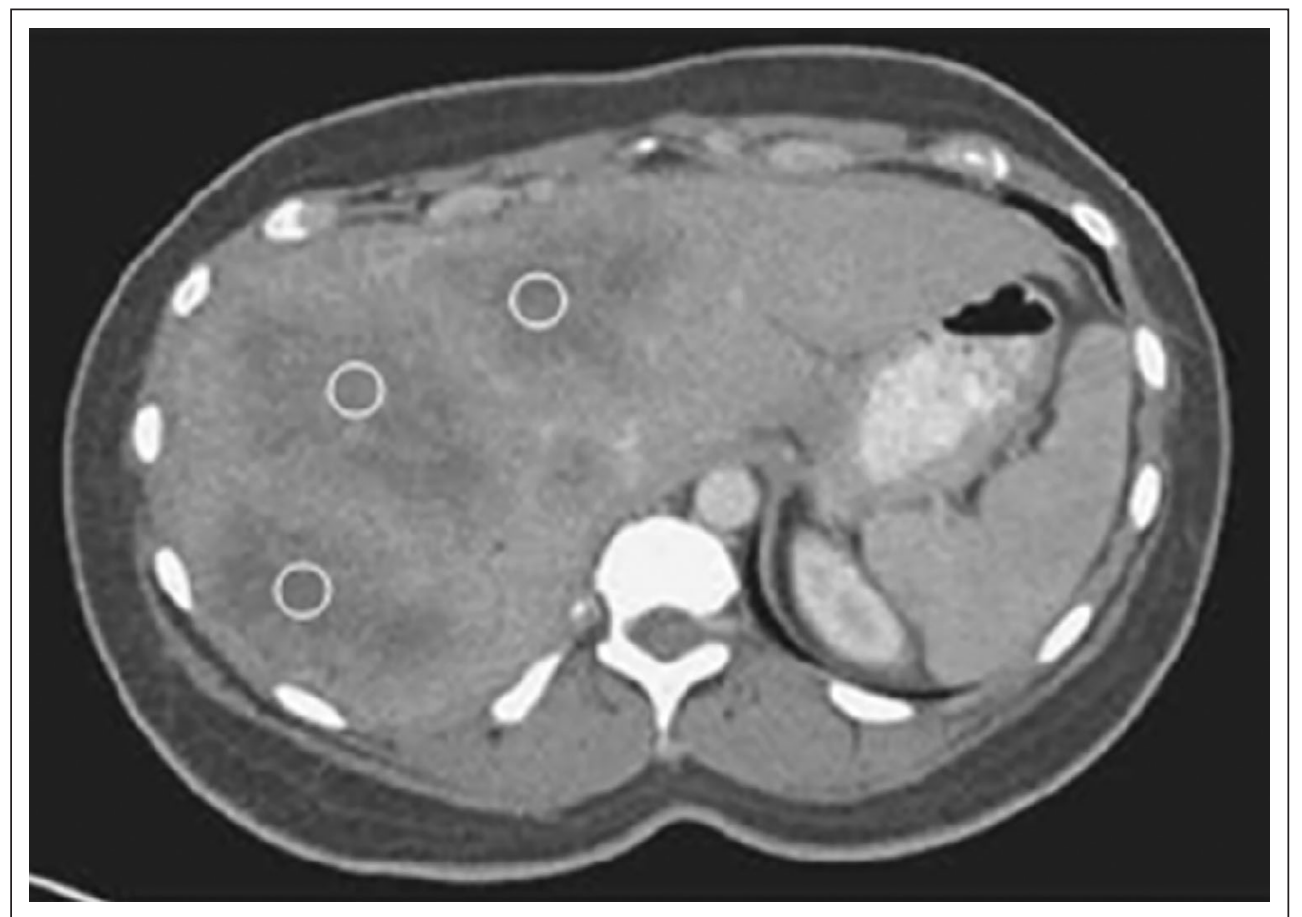

Fig. 2. The placement of ROIs on a CT image of multiple liver lesions.

ROI $\left(\mathrm{HU}_{\mathrm{ROI}}\right)$. The image noise values were obtained from the measurement of the standard deviation of the ROI $\left(\mathrm{SD}_{\mathrm{ROI}}\right)$. The SNR values were calculated by using the following equation (Yu et al., 2016):

$$
S N R=\left(H U_{R O I} / S D_{R O I}\right) \quad-\text { Equation } 1
$$

\section{Statistical analysis}

A Shapiro-Wilk test was conducted to determine the normality distribution of mean attenuation value, image noise, and SNR. As the test was not significant $(p>0.05)$ for all those parameters. Thus normality can be assumed. One-way Analysis of Variance (ANOVA) was performed to determine the mean difference of the image quality parameters among those three kernels using Statistical Package for the Social Sciences (SPSS version 21.0) with $p<0.05$ was deemed as statistically significant.

\section{RESULTS AND DISCUSSION}

ANOVA analysis demonstrated no significant difference in attenuation value of the normal liver and liver lesions among the three kernel reconstructions $(p>0.05)$ (Table $1 \&$ Table 2, respectively). Similarly, no significant changes were observed in image noise and SNR in the liver lesions $(p>0.05)$ (Table 2). Nevertheless, image noise and SNR were significantly changed in the normal liver following the application of B20f, B30f, and B40f kernel reconstructions in three-phase CT liver $(p<0.001)$ as shown in Table 1.

This study investigates the effects of applying three reconstruction kernels of the FBP algorithm (smooth, medium smooth and medium) on image quality in 3-phase CT of the liver. Smooth and medium smooth kernels are the most effective reconstruction techniques for abdominal imaging, whereas medium smooth and medium kernels are the standard reconstruction kernel for $\mathrm{CT}$ abdomen (Jang et al., 2011). An appropriate selection of reconstruction kernel would modify the image impression. The smoothness or sharpness of the images is justified based on the diagnostic necessity. Smooth kernels are preferred to depict subtle contrast differences between larger structures while sharper kernels are recommended for detailed structured inspection and reduced blooming from high-density structures (Nieman et al., 2015). The result on images of normal liver demonstrated that smooth kernel group (B20f) led to a slightly higher attenuation value as compared to medium smooth (B30f) and medium (B40f) kernels, but the variances were not significant. As expected, the B20f group demonstrated lower image noise as compared to the B30f and B40f. The present finding is in agreement with the previous studies by Bhosale et al. (2015) and Völgyes et al. (2017) which reported smooth kernel produced a significantly less image noise as compared to medium and sharp kernels. They concluded smooth kernel is the best reconstruction kernel for improved image quality with significantly reduced image noise in the 
Table 1. Image quality parameters using smooth, medium smooth, and medium reconstruction kernels in the normal liver

\begin{tabular}{lccc}
\hline Parameter & Smooth (B20f) kernel & Medium smooth (B30f) kernel & Medium (B40f) kernel \\
\hline Attenuation & $104.87 \pm 16.04$ & $104.63 \pm 16.11$ & $104.47 \pm 16.11$ \\
Image noise & $11.37 \pm 1.85$ & $13.65 \pm 1.97$ & $16.12 \pm 2.42^{*}$ \\
SNR & $9.51 \pm 2.28$ & $7.87 \pm 1.83$ & $6.66 \pm 1.55^{*}$ \\
\hline
\end{tabular}

Values are expressed as means \pm SD $(n=43)$. SNR (signal-to-noise-ratio). *Significantly difference, $p<0.001$.

Table 2. Image quality parameters using smooth, medium smooth, and medium reconstruction kernels in the liver lesions

\begin{tabular}{lccr}
\hline Parameter & Smooth (B20f) kernel & Medium smooth (B30f) kernel & Medium (B40f) kernel \\
\hline Attenuation & $33.98 \pm 21.08$ & $34.55 \pm 20.88$ & $34.09 \pm 20.03$ \\
Image noise & $12.42 \pm 6.23$ & $14.65 \pm 6.38$ & $17.12 \pm 6.72$ \\
SNR & $3.37 \pm 3.07$ & $2.75 \pm 2.21$ & $2.29 \pm 1.81$ \\
\hline
\end{tabular}

Values are expressed as means \pm SD $(n=20)$. SNR (signal-to-noise-ratio).

CT liver. Yu et al. (2015) claimed the B20f kernel was smoother than B40f in the assessment on a lowcontrast phantom. Reduced image noise using smooth kernel and correspondingly correlated with increased SNR would be justified due to the preservation of attenuation values (Korn et al., 2012)

Furthermore, the SNR of the smooth kernel (B20f) in the normal liver images was found to be significantly higher as compared to groups of medium smooth (B30f) and medium (B40f) kernels. This finding is comparable to a study by Jang et al. (2011) who reported that the highest SNR and peak signal-to-noise ratio (PSNR) values resulted from the smooth kernel. They claimed B20f and B30f kernels were the most effective reconstruction techniques for abdominal $\mathrm{CT}$ images. The result of this study confirmed that the use of smooth reconstruction kernel is the preferred method in image reconstruction technique for the visualization of liver tissues as this reconstruction kernel enhanced the CT image quality by the increased SNR.

The result on CT images of liver lesions found that the medium smooth kernel group (B30f) has a slightly higher mean attenuation value as compared to smooth and medium kernels. For image noise, the smooth kernel group has the lowest mean value as compared to the medium smooth and medium kernel groups. A study by Jang et al. (2011) showed that the detection of small low-contrast lesions was improved as the kernel became smoother. The smooth kernel has been recommended for the assessment of abdominal soft tissue such as a liver lesion or tumor (Jeong et al., 2019).

The effect of image noise on diagnostic accuracy depends on clinical requirements. High image noise is acceptable in abdominal studies with intrinsic high contrast between tissues such as examination of renal stone. However, noisy images can alter the diagnostic performance of CT in studies that entail low contrast between normal and abnormal tissues, such as examination for the assessment of abdominal metastases (Patino et al., 2015). Thus, low image noise is significant in the assessment of the liver and detection of the lesion in liver tissue. It is also important to note that noise in $\mathrm{CT}$ images depends on the number of detected photons, dose level, patient size, slice thickness, algorithm, and electronic noise (Jang et al., 2011). As the FBP algorithm is limited with increased noise, thus the application of a smooth kernel would reduce this limitation. Meanwhile, the iterative reconstruction algorithm reduces the noise level in the $\mathrm{CT}$ image and medium noise suppression is preferable to improve the image quality (Yu et al., 2015).

CT number linearity is one of the most important image quality features in this modality. The mean attenuation values or CT numbers are measured in Hounsfield units (HU). A scanner can map the same physical object into slightly different CT numbers depending on the spectrum of the $x$-ray tube, reconstruction kernel, or correction algorithms (Völgyes et al., 2017), such as a dedicated beam hardening correction (Yu et al., 2015). CT numbers are directly used in diagnostic imaging. Hence, it is of utmost importance that these values are accurate. Furthermore, SNR is another important parameter in low contrast detectability SNR is the ratio of the signal mean or CT number to its standard deviation, and it determines the amount of 
information that can be derived from a measurement (Jang et al., 2011). This parameter is a useful tools with the potential for improving image quality assessment in CT.

In our result, the differences in image noise and SNR values among the reconstruction kernels in the liver lesions were not as significant as in the normal liver. This finding contradicts with a study by Jang et al. (2011). The variance characteristics of the phantom in their study and true lesion in the present study may contribute to the inconsistency of the findings. In our results, the CT images of normal liver tissue were significantly more tolerant with B20f reconstruction kernels than those of B30f and B40f kernels. A smooth (B20f) reconstruction kernel is useful for gaining the best image quality and depicting the spatial resolution of liver CT scanning. Reconstruction kernels are important in the processing of high-quality CT scanning. However, our results showed that the application of these reconstruction kernels did not significantly affect the interpretation of liver lesions. There were a few lesions located in proximity to blood vessels or other normal anatomic features, however, these lesions did not superimpose on top of those features. Some images of liver CT in this study were presented with a single lesion. Most of the lesions were small and this factor led to the variance size of ROIs between the normal liver and liver lesion. The different positions and the size of ROIs for liver lesions may affect the value of mean CT number (Völgyes et al., 2017). This study is limited with a small sample size from a single health institution. Moreover, further investigation using different kernels and algorithms in various CT examinations is recommended.

\section{CONCLUSION}

Application of smooth (B20f), medium smooth (B30f), and medium (B40f) kernel reconstructions would significantly affect the image noise and SNR in the normal liver of CT images instead of liver lesions. Hence, proper selection of reconstruction kernel is important in $\mathrm{CT}$ images reconstruction to improve precision in diagnostic CT interpretation.

\section{ACKNOWLEDGEMENTS}

The authors would like to thank the Department of Radiology, Kuantan Medical Centre, Pahang for the permission and assistance on data collection during this study.

\section{REFERENCES}

Alsleem, H. \& Davidson, R. 2013. Factors affecting contrast-detail performance in computed tomography: a review. Journal of Medical Imaging and Radiation Sciences, 44(2): 62-70.

Bhosale, P., Wagner-Bartak, N., Wei, W., Kundra, V. \& Tamm, E. 2015. Comparing CNR, SNR, and image quality of CT images reconstructed with soft kernel, standard kernel, and standard kernel plus ASIR 30\% techniques. International Journal of Radiology, 2(2): 60-65.

Gervaise, A., Osemont, B., Louis, M., Lecocq, S., Teixeira, P. \& Blum, A. 2014. Standard dose versus low-dose abdominal and pelvic CT: Comparison between filtered back projection versus adaptive iterative dose reduction 3D. Diagnostic and Interventional Imaging, 95(1): 47-53.

Jang, K., Kweon, D.C., Lee, J.W., Choi, J., Goo, E.H., Dong, K.R., Lee. J.S., Jin, G.H. \& Seo, S. 2011. Measurement of image quality in CT images reconstructed with different kernels. Journal of the Korean Physical Society, 58(2): 334-342.

Jensen, K., Martinsen, A.C.T., Tingberg, A., Aaløkken, T.M. \& Fosse, E. 2014. Comparing five different iterative reconstruction algorithms for computed tomography in an ROC study. European Radiology, 24(12): 2989-3002.

Jeong, D., Lee, S., Kim, J., Huh, K., Yi, W., Heo, M. \& Choi, S. 2019. Effects of energy level, reconstruction kernel, and tube rotation time on Hounsfield units of hydroxyapatite in virtual monochromatic images obtained with dualenergy CT. Imaging Science in Dentistry, 49(4): 273-279.

Korn, A., Fenchel, M., Bender, B., Danz, S., Hauser, T.K., Ketelsen, D., Flohr, T., Claussen, C.D., Heuschmid, M., Ernemann, U. \& Brodoefel, H. 2012. Iterative reconstruction in head $\mathrm{CT}$ : Image quality of routine and low-dose protocols in comparison with standard filtered back-projection. American Journal of Neuroradiology, 33(2): 218-224.

Nieman, K., Coenan, A. \& Dijkshoorn, M. 2015. Computed Tomography. In: Advanced Cardiac Imaging. K. Nieman, O. Oliver Gaemperli, P. Lancellotti \& S. Plein (Eds.). Woodhead Publishing. pp. 97-125.

Patino, M., Fuentes, J.M., Singh, S., Hahn, P.F. \& Sahani, D.V. 2015. Iterative reconstruction techniques in abdominopelvic CT: Technical concepts and clinical implementation. American Journal of Roentgenology, 205(1): W19-31. 
Sagara, Y., Hara, A.K., Pavlicek, W., Silva, A.C., Paden, R.G. \& Wu, Q. 2010. Abdominal CT: Comparison of low-dose CT with adaptive statistical iterative reconstruction and routinedose CT with filtered back projection in 53 patients. American Journal of Roentgenology, 195(3): 713-719.

Scharf, M., Brendel, S., Melzer, K., Hentschke, C., May, M., Uder, M. \& Lell, M.M. 2017. Image quality, diagnostic accuracy, and potential for radiation dose reduction in thoracoabdominal CT, using Sinogram Affirmed Iterative Reconstruction (SAFIRE) technique in a longitudinal study. PLOS ONE, 12(7): e 0180302.

Seeram, E. 2015. Computed Tomography: Physical Principles, Clinical Applications and Quality Control. 4th Ed. Saunders, USA.

Staniszewska, M. \& Chrusciak, D. 2017. Iterative reconstruction as a method for optimisation of computed tomography procedures. Polish Journal of Radiology, 82: 792-797.

Vardhanabhuti, V., Ilyas, S., Gutteridge, C., Freeman, S.J. \& Roobottom, C.A. 2013. Comparison of image quality between filtered back-projection and the adaptive statistical and novel modelbased iterative reconstruction techniques in abdominal CT for renal calculi. Insights into Imaging, 4(5): 661-669.
Völgyes, D., Pedersen, M., Stray-Pedersen, A., Waaler, D. \& Martinsen, A.C. 2017. How different iterative and filtered back projection kernels affect computed tomography numbers and low contrast detectability. Journal of Computer Assisted Tomography, 41(1): 75-81.

Yu, K., Yi-Yang, L., Rheun-Chuan, L., Chung-Jung, L., Yi-You, C. \& Wan-You, G. 2016. Comparison of image quality from filtered back projection, statistical iterative reconstruction, and modelbased iterative reconstruction algorithms in abdominal computed tomography. Medicine, 95(31): e4456.

Yu, L. \& Leng, S. 2016. Image reconstruction techniques [WWW Document]. URL https:// www.imagewisely.org/Imaging-Modalities/ Computed-Tomography/Image-ReconstructionTechniques (accessed 4.23.20).

Yu, L., Vrieze, T.J., Leng, S., Fletcher, J.G. \& Mccollough, C.H. 2015. Technical note: Measuring contrast and noise-dependent spatial resolution of an iterative reconstruction method in CT using ensemble averaging. Medical Physics, 42(5): 2261-2267. 